Fixed Point Theory, 18(2017), No. 2, 689-702

DOI 10.24193/fpt-ro.2017.2.55

http://www.math.ubbcluj.ro/ nodeacj/sfptcj.html

\title{
A GENERALIZATION OF ISTRĂŢESCU'S FIXED POINT THEOREM FOR CONVEX CONTRACTIONS
}

\author{
RADU MICULESCU* AND ALEXANDRU MIHAIL** \\ University of Bucharest, Faculty of Mathematics and Computer Science \\ Str. Academiei 14, 010014 Bucharest, Romania \\ E-mail: *miculesc@yahoo.com, ${ }^{* *}$ mihail_alex@yahoo.com
}

\begin{abstract}
In this paper we prove a generalization of Istrăţescu's theorem for convex contractions. More precisely, we introduce the concept of iterated function system consisting of convex contractions and prove the existence and uniqueness of the attractor of such a system. In addition we study the properties of the canonical projection from the code space into the attractor of an iterated function system consisting of convex contractions.
\end{abstract}

Key Words and Phrases: Convex contractions, fixed points, iterated function systems consisting of convex contractions.

2010 Mathematics Subject Classification: 54H25, 28A80, 47H10.

\section{REFERENCES}

[1] J. Andres, M. Rypka, Multivalued fractals and hyperfractals, Internat. J. Bifur. Chaos Appl. Sci. Engrg., 22(2012), 27 pp.

[2] M. Boriceanu, M. Bota, A. Petruşel, Multivalued fractals in b-metric spaces, Cent. Eur. J. Math., 8(2010), 367-377.

[3] C. Chifu, A. Petruşel, Multivalued fractals and generalized multivalued contractions, Chaos Solitons Fractals, 36(2008), 203-210.

[4] D. Dumitru, Generalized iterated function systems containing Meir-Keeler functions, An. Univ. Bucureşti, Mat., 58(2009), 109-121.

[5] V. Istrăţescu, Some fixed point theorems for convex contraction mappings and convex nonexpansive mappings (I), Libertas Math., 1(1981), 151-164.

[6] V. Istrățescu, Some fixed point theorems for convex contraction mappings and mappings with convex diminishing diameters - I, Annali di Mat. Pura Appl., 130(1982), 89-104.

[7] V. Istrăţescu, Some fixed point theorems for convex contraction mappings and mappings with convex diminishing diameters - II, Annali di Mat. Pura Appl., 134(1983), 327-362.

[8] V. Ghorbanian, S. Rezapour, N. Shahzad, Some ordered fixed point results and the property (P), Comput. Math. Appl., 63(2012), 1361-1368.

[9] G. Gwóźdź-Łukowska, J. Jachymski, IFS on a metric space with a graph structure and extensions of the Kelisky-Rivlin theorem, J. Math. Anal. Appl., 356(2009), 453-463.

[10] N. Hussain, M.A. Kutbi, S. Khaleghizadeh, P. Salimi, Discussions on recent results for $\alpha-\Psi-$ contractive mappings, Abstr. Appl. Anal., vol. 2014, Article ID 456482, 13 pages, 2014.

[11] M. Klimek, M. Kosek, Generalized iterated function systems, multifunctions and Cantor sets, Ann. Polon. Math., 96(2009), 25-41. 
[12] A. Latif, W. Sintunavarat, A. Ninsri, Approximate fixed point theorems for partial generalized convex contraction mappings in $\alpha$-complete metric spaces, Taiwanese J. Math., 19(2015), 315333.

[13] K. Leśniak, Infinite iterated function systems: a multivalued approach, Bull. Pol. Acad. Sci. Math., 52(2004), 1-8.

[14] E. Llorens-Fuster, A. Petruşel, J.-C. Yao, Iterated function systems and well posedness, Chaos Solitons Fractals, 41(2009), 1561-1568.

[15] L. Máté, The Hutchinson-Barnsley theory for certain noncontraction mappings, Period. Math. Hungar., 27(1993), 21-33.

[16] M.A. Miandaragh, M. Postolache, S. Rezapour, Approximate fixed points of generalized convex contractions, Fixed Point Theory Appl., vol. 2013, article 255, 2013.

[17] A. Mihail, R. Miculescu, Applications of fixed point theorems in the theory of generalized IFS, Fixed Point Theory Appl., 2008, Art. ID 312876, 11 pp.

[18] A. Mihail, R. Miculescu, The shift space for an infinite iterated function system, Math. Reports, 61(2009), 21-31.

[19] A. Mihail, R. Miculescu, Generalized IFSs on noncompact spaces, Fixed Point Theory Appl., 2010, Art. ID 584215, 15 pp.

[20] A. Petruşel, Iterated function system of locally contractive operators, Rev. Anal. Numér. Théor. Approx., 33(2004), 215-219.

[21] A. Petruşel, Fixed point theory and the mathematics of fractals, Topics in Mathematics, Computer Science and Philosophy, Presa Univ. Clujeană, Cluj-Napoca, 2008, 165-172.

[22] N.A. Secelean, Iterated function systems consisting of F-contractions, Fixed Point Theory Appl., 2013, 2013:277.

[23] N.A. Secelean, Countable Iterated Function Systems, Lambert Academic Publishing, 2013.

[24] N.A. Secelean, Generalized iterated function systems on the space $l^{\infty}(X)$, J. Math. Anal. Appl., 410(2014), 847-458.

[25] F. Strobin, J. Swaczyna, On a certain generalization of the iterated function system, Bull. Australian Math. Soc., 87(2013), 37-54.

[26] F. Strobin, Attractors of generalized IFSs that are not attractors of IFSs, J. Math. Anal. Appl., 422(2015), 99-108.

Received: May 14, 2015; Accepted: February 20, 2016. 
